Supplement of Hydrol. Earth Syst. Sci., 23, 3571-3591, 2019

https://doi.org/10.5194/hess-23-3571-2019-supplement

(c) Author(s) 2019. This work is distributed under

the Creative Commons Attribution 4.0 License.

(c) (1)
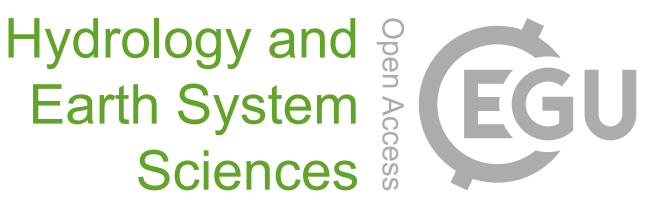

Supplement of

\title{
Assessing inter-annual and seasonal patterns of DOC and DOM quality across a complex alpine watershed underlain by discontinuous permafrost in Yukon, Canada
}

Nadine J. Shatilla and Sean K. Carey

Correspondence to: Nadine J. Shatilla (n.j.shatilla@gmail.com)

The copyright of individual parts of the supplement might differ from the CC BY 4.0 License. 


\begin{tabular}{|c|c|c|c|c|c|c|c|c|c|c|c|c|c|}
\hline \multirow[t]{2}{*}{ Year } & \multirow[t]{2}{*}{ Sites } & \multicolumn{3}{|l|}{$\mathrm{DOC}(\mathrm{mg} / \mathrm{L})$} & \multicolumn{3}{|l|}{ SUVA254 } & \multicolumn{3}{|l|}{ BIX } & \multicolumn{3}{|l|}{ FI } \\
\hline & & Spring & Summer & F/W & Spring & Summer & F/W & Spring & Summer & F/W & Spring & Summer & F/W \\
\hline \multicolumn{14}{|l|}{2015} \\
\hline & $\mathrm{BB}$ & $1.77 \pm 0.66(5)$ & $1.25 \pm 0.31(11)$ & $1.19 \pm 0.39(9)$ & $3.30 \pm 0.58(3)$ & $3.88 \pm 0.55(4)$ & $2.72 \pm 0.42(2)$ & $0.55 \pm 0.04$ & $0.60 \pm 0.01$ & $0.60 \pm 0.03$ & $1.49 \pm 0.06$ & $1.54 \pm 0.03$ & $1.57 \pm 0.76$ \\
\hline & CL & $2.90(1)$ & & $2.60(1)$ & & & & & & & & & \\
\hline & GC & $2.89 \pm 2.29(52)$ & $1.07 \pm 0.21(36)$ & $1.75 \pm 0.76(17)$ & $3.21 \pm 0.84(22)$ & $3.81 \pm 0.75(14)$ & $2.42 \pm 0.33(3)$ & $0.69 \pm 0.06$ & $0.60 \pm 0.02$ & $0.65 \pm 0.02$ & $1.52 \pm 0.06$ & $1.54 \pm 0.04$ & $1.57 \pm 0.04$ \\
\hline & W1 & $15.8(1)$ & & & $3.94(1)$ & & & $0.47(1)$ & & & $1.46(1)$ & & \\
\hline & WCO & & & $1.79 \pm 0.90(14)$ & & & $2.21 \pm 0.17(10)$ & & & $0.66 \pm 0.03$ & & & $1.62 \pm 0.03$ \\
\hline \multirow{6}{*}{2016} & & & & & & & & & & & & & \\
\hline & $\mathrm{BB}$ & $2.34 \pm 0.87(14)$ & $1.42 \pm 0.27(23)$ & $1.50 \pm 0.20(3)$ & $3.56 \pm 0.48(10)$ & $2.81 \pm 0.59(16)$ & $2.45 \pm 0.28(3)$ & $0.513 \pm 0.03$ & $0.58 \pm 0.03$ & $0.62 \pm 0.02$ & $1.45 \pm 0.04$ & $1.52 \pm 0.06$ & $1.52 \pm 0.00$ \\
\hline & CL & & $3.15 \pm 0.35(2)$ & & & & & & & & & & \\
\hline & GC & $4.32 \pm 2.56(43)$ & $1.71 \pm 0.34(32)$ & $2.00 \pm 0.57(20)$ & $3.86 \pm 1.40(37)$ & $2.86 \pm 0.38(17)$ & $3.14 \pm 0.32(11)$ & $0.513 \pm 0.06$ & $0.58 \pm 0.03$ & $0.60 \pm 0.04$ & $1.45 \pm 0.04$ & $1.50 \pm 0.04$ & $1.50 \pm 0.02$ \\
\hline & W1 & $6.70(1)$ & $7.37 \pm 0.64(10)$ & $6.95 \pm 0.21(2)$ & $4.77(1)$ & $4.04 \pm 0.60(7)$ & & $0.58(1)$ & $0.63 \pm 0.05$ & & $1.58(1)$ & $1.54 \pm 0.04$ & \\
\hline & WCO & $2.69 \pm 0.80(18)$ & $2.58 \pm 0.44(22)$ & $2.35 \pm 0.35(3)$ & $2.83 \pm 0.42(12)$ & $2.70 \pm 0.28(19)$ & $2.69 \pm 0.11(2)$ & $0.582 \pm 0.04$ & $0.60 \pm 0.02$ & $0.66 \pm 0.03$ & $1.53 \pm 0.02$ & $1.54 \pm 0.03$ & $1.55 \pm 0.01$ \\
\hline \multirow[t]{5}{*}{2017} & $\mathrm{BB}$ & $2.70(1)$ & $2.17 \pm 0.45(7)$ & $1.15 \pm 0.14(6)$ & & $3.02 \pm 0.38(6)$ & $3.15 \pm 0.17(6)$ & & $0.55 \pm 0.03$ & $0.61 \pm 0.02$ & & $1.48 \pm 0.03$ & $1.55 \pm 0.02$ \\
\hline & $\mathrm{CL}$ & & $3.23 \pm 0.15(4)$ & $2.96 \pm 0.09(5)$ & & $2.66 \pm 0.15(3)$ & $2.82 \pm 0.08(5)$ & & $0.66 \pm 0.03$ & $0.69 \pm 0.02$ & & $1.48 \pm 0.01$ & $1.50 \pm 0.03$ \\
\hline & $\mathrm{GC}$ & $3.15 \pm 0.64(2)$ & $2.33 \pm 0.71(7)$ & $1.28 \pm 0.18(6)$ & & $3.14 \pm 0.23(5)$ & & & $0.55 \pm 0.03$ & & & $1.45 \pm 0.03$ & \\
\hline & W1 & & & $6.10(1)$ & & & $5.26(1)$ & & & $0.62(1)$ & & & $1.66(1)$ \\
\hline & WCO & $4.42 \pm 1.84(6)$ & $2.72 \pm 0.37(5)$ & $2.14 \pm 0.11(5)$ & & $2.83 \pm 0.25(3)$ & $2.94 \pm 0.10(3)$ & & $0.61 \pm 0.03$ & $0.64 \pm 0.01$ & & $1.51 \pm 0.02$ & $1.57 \pm 0.02$ \\
\hline
\end{tabular}

Table S1. This table is similar to Table 1 in the manuscript but incorporates all samples used for principal component analysis (PCA). Additional sites (CL, W1) and additional years of data for sites BB, GC and WCO were used in the analysis to investigate influence of landscape type. Notation: Mean \pm standard deviation (number of samples). 


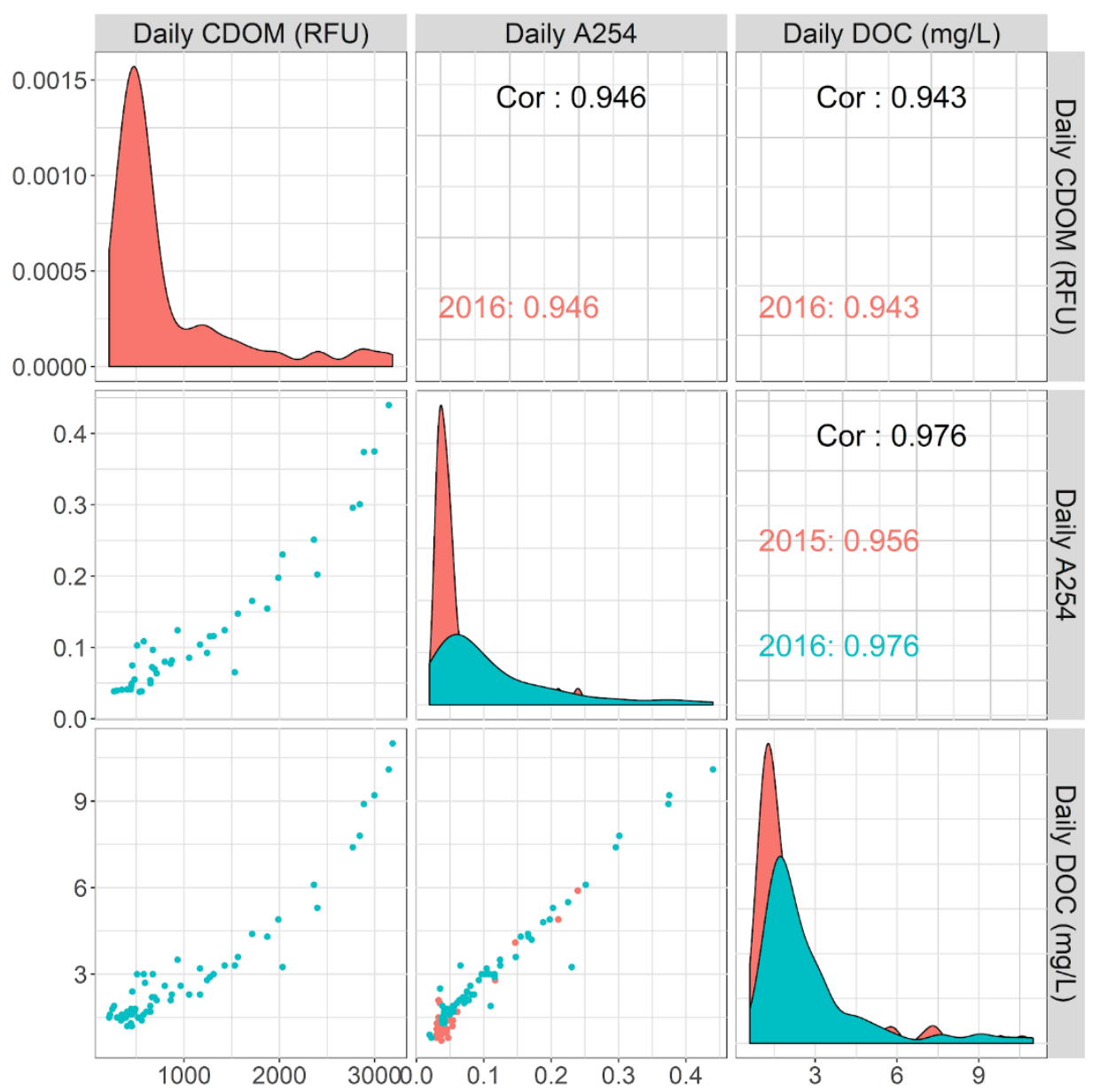

Figure S1. Correlation matrices of average daily CDOM (RFU), A254 ( $\left.\mathrm{nm}^{-1}\right)$ and DOC concentration $\left(\mathrm{mg} \mathrm{L}^{-1}\right)$. No CDOM was measure in 2015 so it was not possible to separate out that year. Correlation was calculated using Pearson at 95\% significance level ( $\mathrm{p}<0.001$ in all cases). 


\begin{tabular}{|c|c|c|c|c|c|}
\hline Year Site & Spring $\left(\mathrm{R}^{2}\right)$ & Summer $\left(\mathrm{R}^{2}\right)$ & $1\left(\mathrm{R}^{2}\right)$ & Spring \& Summer $\left(\mathrm{R}^{2}\right)$ & Spring, Summer \& Fall $\left(\mathrm{R}^{2}\right)$ \\
\hline $2002 G C$ & 0.047 & 0.180 & & 0.063 & \\
\hline $2003 G C$ & 0.041 & 0.001 & & 0.034 & \\
\hline $2006 G C$ & 0.021 & 0.175 & & 0.029 & \\
\hline $2008 G C$ & 0.024 & 0.215 & & 0.005 & \\
\hline $2015 G C$ & 0.263 & 0.004 & 0.547 & 0.316 & 0.314 \\
\hline $2016 G C$ & 0.115 & 0.536 & 0.551 & 0.039 & 0.048 \\
\hline $2016 \mathrm{WCO}$ & 0.066 & 0.783 & & 0.010 & 0.011 \\
\hline
\end{tabular}

Table S2. Regressions between discharge (Q) and DOC concentrations (C) were performed using the CQregression function in the RiverLoad package (Nava et al., 2019) for GC in 2002, 2003, 2006, 2008, 2015 and 2016. A statistically significant correlation between $\mathrm{C}$ and $\mathrm{Q}$ was necessary to perform the regression. 
Kaiser-Meyer-Olkin factor

Overall MSA $=0.77$

MSA for each item:

$\mathrm{FI}=0.98$

Fresh $=0.68$

HIX $=0.86$

$\mathrm{BIX}=0.69$

SUVA $=0.91$

$\mathrm{DOC}=0.94$

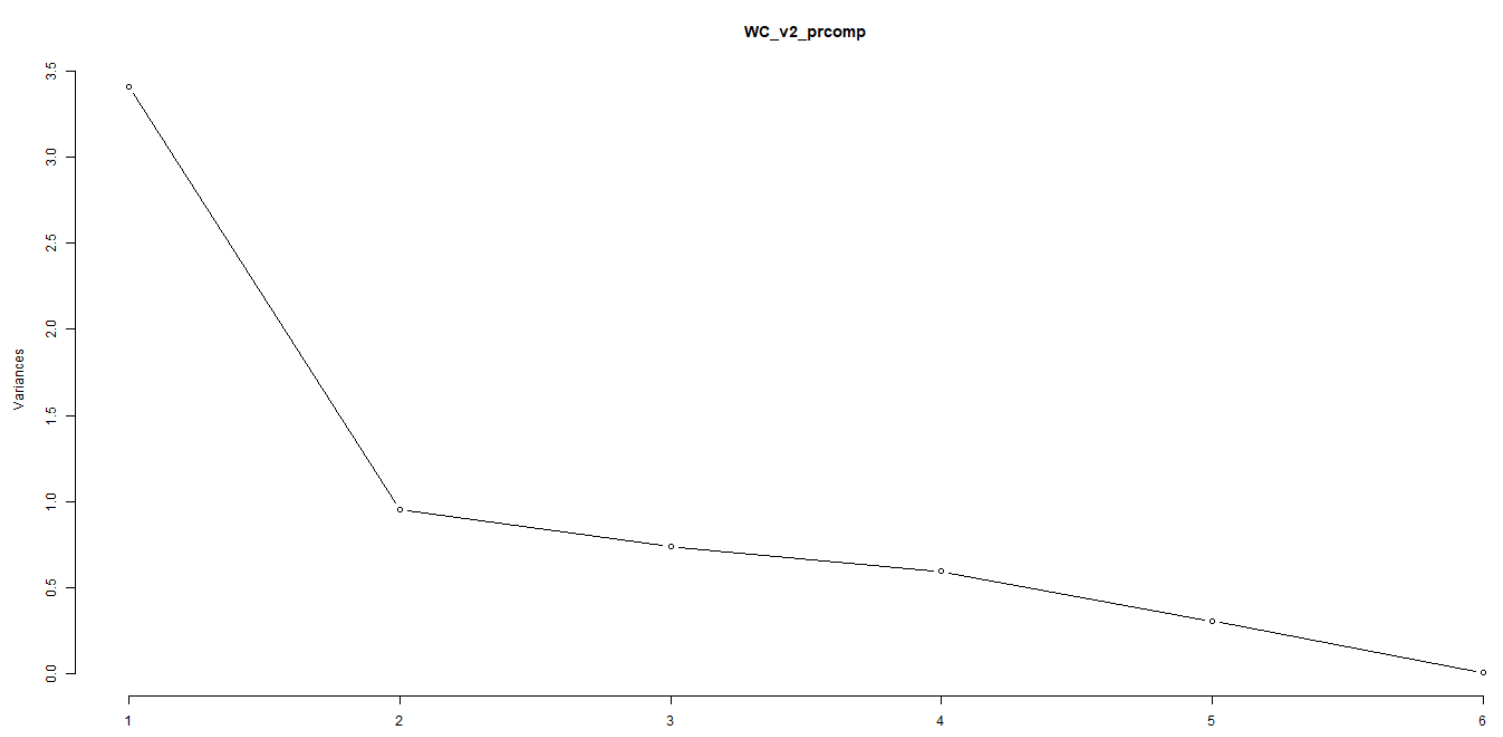

Figure S2. Scree plot for PCA. 


\begin{tabular}{lllll}
\hline & PC1 & PC2 & PC3 & PC4 \\
\hline Standard dev & 1.8472 & 0.9747 & 0.8577 & 0.7702 \\
Proportion of variance & 0.5687 & 0.1583 & 0.1226 & 0.09887 \\
Cumulative proportion & 0.5687 & 0.7270 & 0.8496 & 0.9485 \\
\hline
\end{tabular}

Table S3. Standard deviation, proportion of variance explained by each PC (x100 for

$\%)$ and cumulative proportion explained.
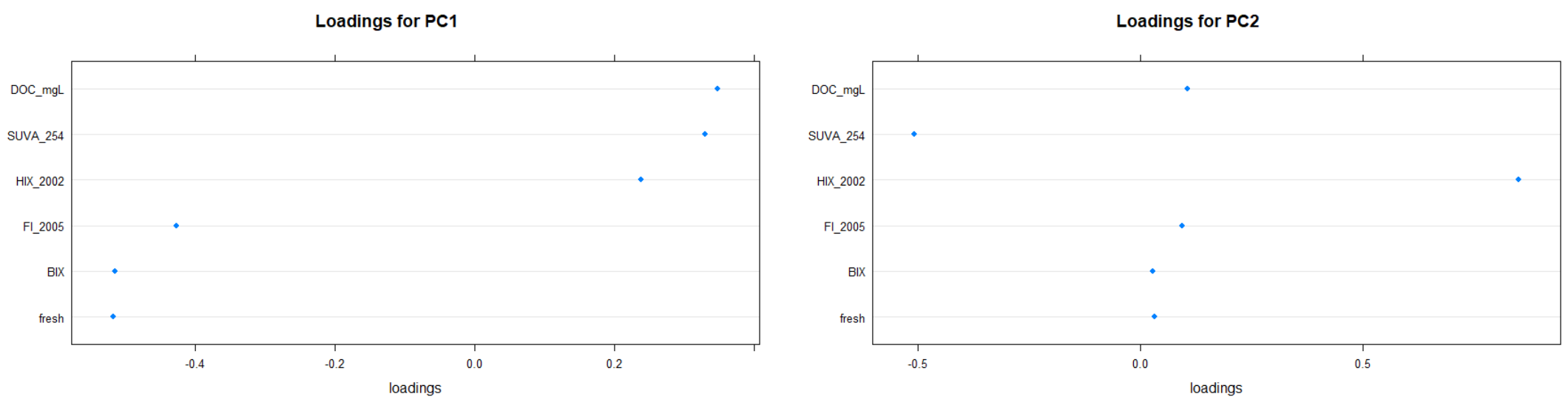

Figure S3. Dot plots of loadings per PC in PCA. 